CRYSTALLOGRAPHIC COMMUNICATIONS

ISSN 2056-9890

Received 31 July 2017

Accepted 22 August 2017

Edited by P. Bombicz, Hungarian Academy of Sciences, Hungary

Keywords: crystal structure; thiophene; polythiophene; 1,2,4-triazole-3-thione; disorder.

CCDC reference: 1570281

Supporting information: this article has supporting information at journals.iucr.org/e

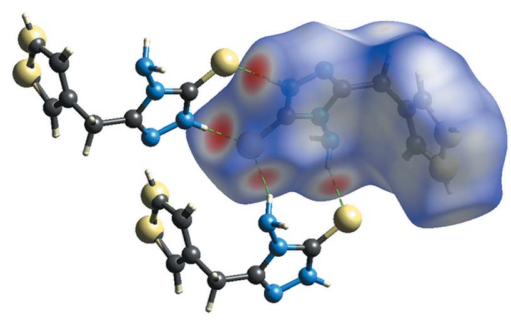

OPEN $\odot$ ACCESS

\section{Crystal structure of 4-amino-3-(thiophen-3-yl- methyl)-1H-1,2,4-triazole-5(4H)-thione}

Trung Vu Quoc, ${ }^{a}$ Linh Nguyen Ngoc, ${ }^{a}$ Vy Do Truc, ${ }^{a}$ Linh Duong Khanh, ${ }^{a}$ Hung Ha Manh, ${ }^{\text {b }}$ Chien Thang Pham ${ }^{c}$ and Luc Van Meervelt ${ }^{\mathrm{d} *}$

${ }^{\mathbf{a}}$ Faculty of Chemistry, Hanoi National University of Education, 136 Xuan Thuy, Cau Giay, Hanoi, Vietnam, ${ }^{\mathbf{b}}$ Faculty of Basic Sciences, University of Mining and Geology, Duc Thang, Bac Tu Liem, Hanoi, Vietnam, ' VNU University of Science, Department of Inorganic Chemistry, 19 Le Thanh Tong Street, Hoan Kiem Discrict, Hanoi, Vietnam, and dDepartment of Chemistry, KU Leuven, Biomolecular Architecture, Celestijnenlaan 200F, Leuven (Heverlee), B-3001, Belgium. *Correspondence e-mail: luc.vanmeervelt@kuleuven.be

In the title compound, $\mathrm{C}_{7} \mathrm{H}_{8} \mathrm{~N}_{4} \mathrm{~S}_{2}$, the thiophene ring shows rotational disorder over two orientations in a 0.6957 (15):0.3043 (15) ratio. The plane of the 1,2,4triazole ring makes a dihedral angle of $75.02(17)^{\circ}$ with the major-disorder component of the thiophene ring. In the crystal, two types of inversion dimers, described by the graph-set motifs $R_{2}^{2}(8)$ and $R_{2}^{2}(10)$, are formed by $\mathrm{N}-\mathrm{H} \cdots \mathrm{S}$ interactions. Chains of molecules running in the [101] direction are linked by weaker $\mathrm{N}-\mathrm{H} \cdots \mathrm{N}$ interactions. The thiophene ring is involved in $\pi-\pi$ and $\mathrm{C}-$ $\mathrm{H} \cdots \pi$ interactions.

\section{Chemical context}

Recently, the synthesis, characterization and antifungal activities, together with crystal structure determinations, of thiophene-based heterocyclic chalcones have been investigated (Ming et al., 2017). Thiophene-containing $\beta$-diketonate complexes of copper(II) have been studied and their deposits obtained by electropolymerization have been characterized (Oyarce et al., 2017). Combinations of the thiophene ring with other heterocyclic rings have also been investigated, such as a $\beta$-keto-enol group embedded with thiophene and pyridine moieties giving interesting applications in the field of solidphase extraction (Radi et al., 2016).

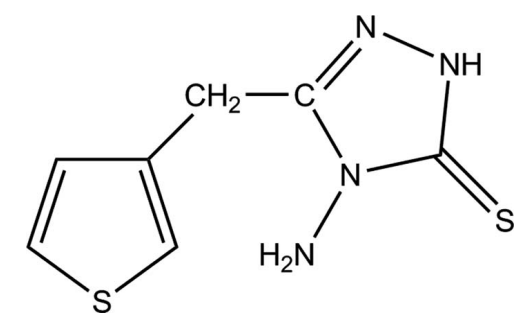

As part of our ongoing studies of new polythiophenes and their properties (Nguyen et al., 2016; Vu et al., 2016; Vu Quoc et al., 2017), we have synthesized a new thiophene monomer containing an additional 1,2,4-triazole ring. The polymer obtained from 4-amino-3-(thiophen-3-ylmethyl)-1 $\mathrm{H}-1,2,4-$ triazole-5(4H)-thione using $\mathrm{FeCl}_{3}$ as oxidant was further characterized by IR and NMR spectroscopy, and TGA and is soluble in most common organic solvents, such as DMF and DMSO. We present here the synthesis and crystal structure of the title compound, $\mathbf{3}$. 
Table 1

Hydrogen-bond geometry $\left(\AA,^{\circ}\right)$.

$\mathrm{Cg} 3$ is the centroid of the $\mathrm{N} 2 / \mathrm{N} 3 / \mathrm{N} 4 / \mathrm{C} 6 / \mathrm{C} 7$ ring.

\begin{tabular}{lllll}
\hline$D-\mathrm{H} \cdots A$ & $D-\mathrm{H}$ & $\mathrm{H} \cdots A$ & $D \cdots A$ & $D-\mathrm{H} \cdots A$ \\
\hline $\mathrm{N} 1-\mathrm{H} 1 C \cdots \mathrm{N} 3^{\mathrm{i}}$ & $0.909(15)$ & $2.622(15)$ & $3.3847(13)$ & $142.0(12)$ \\
$\mathrm{N} 1-\mathrm{H} 1 D \cdots \mathrm{S} 2^{\text {ii }}$ & $0.849(17)$ & $2.628(16)$ & $3.4163(9)$ & $154.9(13)$ \\
$\mathrm{N} 4-\mathrm{H} 4 \cdots \mathrm{S} 2^{\mathrm{iii}}$ & $0.890(16)$ & $2.395(15)$ & $3.2847(9)$ & $178.2(12)$ \\
$\mathrm{C} 1 B-\mathrm{H} 1 B \cdots C g 3^{\text {iv }}$ & 0.95 & 2.78 & $3.503(11)$ & 134 \\
\hline
\end{tabular}

Symmetry codes: (i) $x-\frac{1}{2},-y+\frac{1}{2}, z-\frac{1}{2}$; (ii) $-x+1,-y+2,-z+1$; (iii) $-x,-y+2$, $-z+1 ;$ (iv) $-x+1,-y+2,-z$.

\section{Structural commentary}

The title compound (Fig. 1) crystallizes in the monoclinic space group $P 2_{1} / n$ with one molecule in the asymmetric unit. The thiophene ring is disordered over two orientations in a rotation of approximately $180^{\circ}$ around the $\mathrm{C} 5-\mathrm{C} 3$ bond [occupancy factors $=0.6957$ (15) for ring $A$ or $\mathrm{S} 1 A / \mathrm{C} 1 A / \mathrm{C} 2 A$ / $\mathrm{C} 3 / \mathrm{C} 4 A$ and 0.3043 (15) for ring $B$ or $\mathrm{S} 1 B / \mathrm{C} 1 B / \mathrm{C} 2 B / \mathrm{C} 3 / \mathrm{C} 4 B]$. The 1,2,4-triazole ring is almost planar (r.m.s. deviation = $0.001 \AA$ for ring $\mathrm{N} 2 / \mathrm{N} 3 / \mathrm{N} 4 / \mathrm{C} 6 / \mathrm{C} 7$ ), with the substituents $\mathrm{N} 1$, S2 and C5 deviating by -0.034 (1), 0.008 (1) and 0.093 (1) ̊, respectively. Due to the $s p^{3}$ character of the linking atom C5, the planes of the five-membered rings make dihedral angles of 75.02 (17) (ring $A$ ) and $76.4(4)^{\circ}(\operatorname{ring} B)$, which results in a Vshaped conformation. Atom N1 clearly has an $s p^{3}$ hybridization as shown by the bond angles.

\section{Supramolecular features}

The crystal packing of the title compound is shown in Fig. 2. The 1H-1,2,4-triazole-5(4H)-thione ring possesses an $\mathrm{NH}_{2}$ group, which, in principle, can act as a donor or acceptor for hydrogen bonding, an NH group, which can act as a donor, and an $\mathrm{N}$ atom and $\mathrm{C}=\mathrm{S}$ group, which can only act as acceptors. Two types of inversion dimers are formed (Fig. 3 and Table 1). The first one, described as graph-set motif $R_{2}^{2}(8)$, involves hydrogen bonds between the $\mathrm{NH}$ and $\mathrm{C}=\mathrm{S}$ groups, whereas in the second one, the $\mathrm{NH}_{2}$ group interacts with the $\mathrm{C}=\mathrm{S}$ grouping, resulting in a ring structure of graph-set

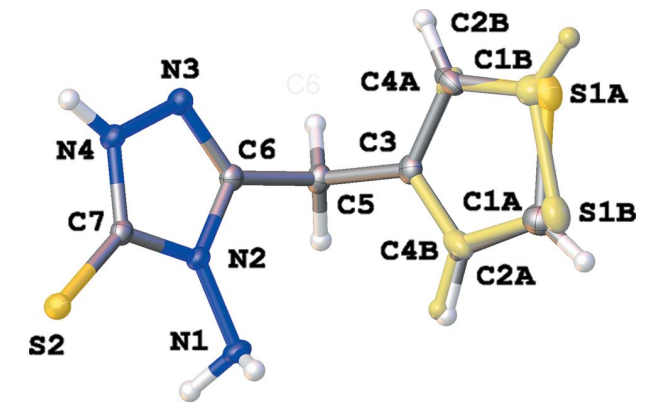

Figure 1

A view of the asymmetric unit of the title compound, showing the atomlabelling scheme. Displacement ellipsoids are drawn at the $50 \%$ probability level. $\mathrm{H}$ atoms are shown as small circles of arbitrary radii. The minor component of the disordered thiophene rings is shown in pale yellow.

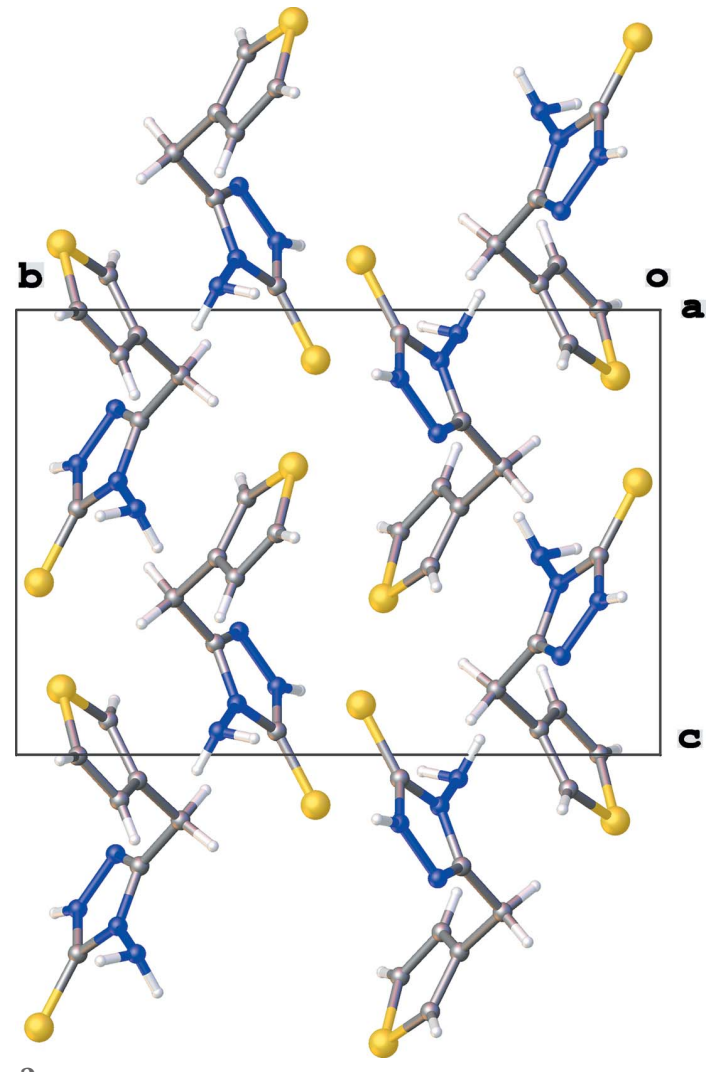

Figure 2

Crystal packing of the title compound shown in projection down the $a$ axis.

$R_{2}^{2}(10)$. The second $\mathrm{H}$ atom of the $\mathrm{NH}_{2}$ group interacts with the $\mathrm{N}$ atom of a neighbouring $1 H$-1,2,4-triazole-5(4H)-thione ring, resulting in chains of graph-set $C(5)$ in the [101] direction (Fig. 3 and Table 1).

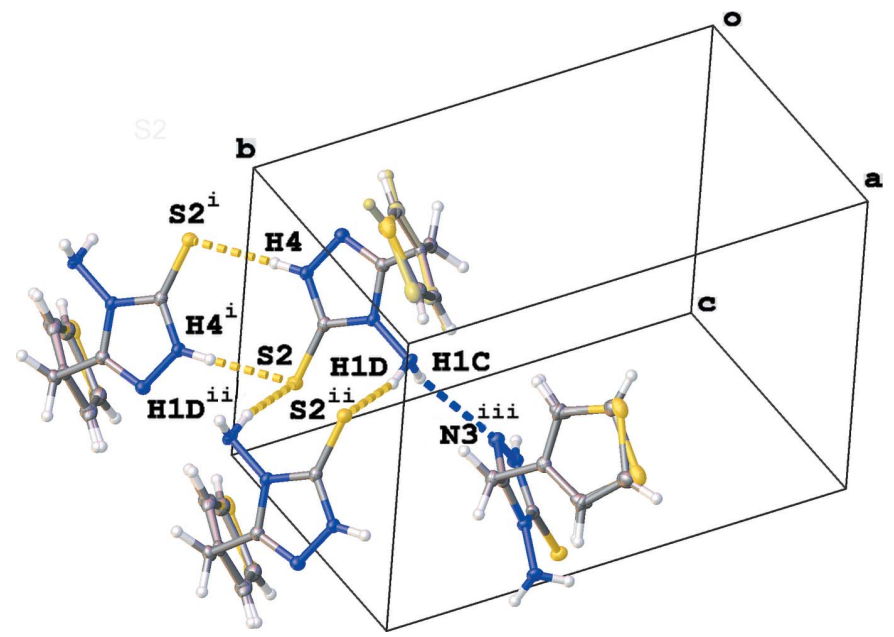

Figure 3

Part of the crystal packing of the title compound, showing the rings of graph-set motif $R_{2}^{2}(8)$ and $R_{2}^{2}(10)$ formed by $\mathrm{N}-\mathrm{H} \cdots \mathrm{S}$ hydrogen-bond interactions [see Table 1 ; symmetry codes: (i) $-x,-y+2,-z+1$; (ii) $-x+1,-y+2,-z+1$; (iii) $\left.x+\frac{1}{2},-y+\frac{3}{2}, z+\frac{1}{2}\right]$ and a chain of graph-set motif $C(5)$. 


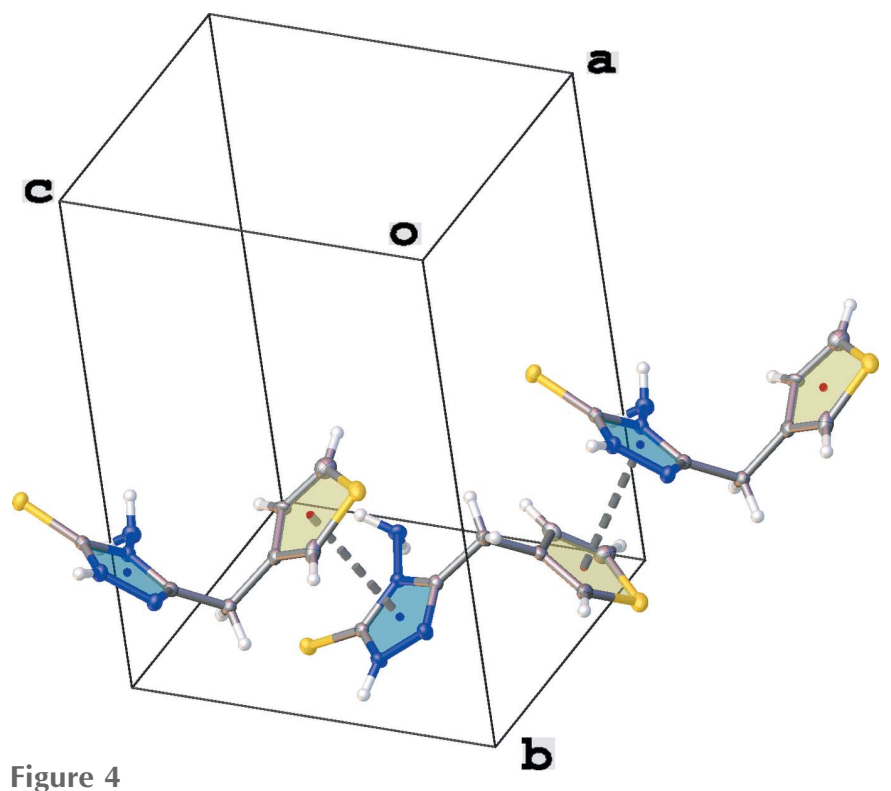

Part of the crystal packing of the title compound, showing the $\pi-\pi$ stacking interactions between the thiophene (yellow) and 1,2,4-triazole (blue) rings (only the major component of the disordered thiophene ring is shown).

The disordered thiophene ring is only involved in a $\pi-\pi$ stacking interaction with the 1,2,4-triazole ring $\left[C g 1 \cdots C g 3^{\mathrm{i}}=\right.$ 3.415 (2) $\AA$ and $C g 2 \cdots C g 3^{\mathrm{i}}=3.440$ (5) $\AA$; $C g 1, C g 2$ and $C g 3$ are the centroids of ring $A$, ring $B$ and the 1,2,4-triazole ring, respectively; symmetry code: (i) $x+\frac{1}{2},-y+\frac{3}{2}, z-\frac{1}{2}$; Fig. 4]. The crystal packing shows a weak $\mathrm{C}-\mathrm{H} \cdots \pi$ interaction (Table 1 ) and contains no voids.

The packing was further investigated by an analysis of the Hirshfeld surface and two-dimensional fingerprint plots using CrystalExplorer (McKinnon et al., 2007; Spackman \& Jayatilaka, 2009). The donors and acceptors corresponding to the $\mathrm{N}-\mathrm{H} \cdots \mathrm{S}$ interactions are visible as bright-red spots in Fig. 5(a). The pale-red spots in Fig. 5(b) are the weaker $\mathrm{N}-$ $\mathrm{H} \cdots \mathrm{N}$ and $\mathrm{C}-\mathrm{H} \cdots \mathrm{N}$ interactions. The relative contributions of the different intermolecular interactions to the Hirshfeld surface area in descending order are: $\mathrm{H} \cdots \mathrm{H}(40.4 \%), \mathrm{S} \cdots \mathrm{H}$ $(26.7 \%), \mathrm{N} \cdots \mathrm{H}(13.3 \%), \mathrm{C} \cdot \mathrm{H}(8.2 \%), \mathrm{C} \cdots \mathrm{C}(4.1 \%), \mathrm{C} \cdots \mathrm{N}$ $(3.7 \%), \mathrm{S} \cdots \mathrm{C}(2.3 \%)$ and $\mathrm{S} \cdots \mathrm{N}(1.2 \%)$. This illustrates that the weak $\mathrm{N}-\mathrm{H} \cdots \mathrm{N}$ and $\mathrm{C}-\mathrm{H} \cdots \mathrm{N}$ interactions contribute significantly to the packing of the title compound.

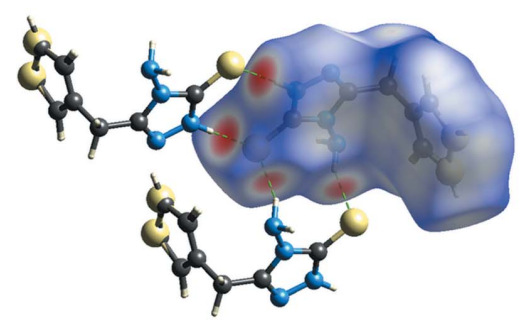

(a)

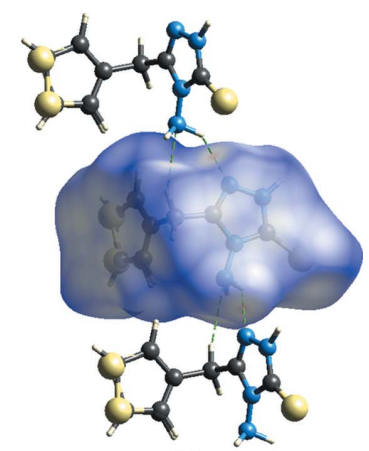

(b)

Figure 5

Hirshfeld surface for title compound mapped over $d_{\text {norm }}$ over the range -0.436 to 1.179 a.u., highlighting $(a)$ the $\mathrm{N}-\mathrm{H} \cdots \mathrm{S}$ hydrogen bonding and (b) the $\mathrm{N}-\mathrm{H} \cdots \mathrm{N}$ and $\mathrm{C}-\mathrm{H} \cdots \mathrm{N}$ interactions.

\section{Database survey}

A search of the Cambridge Structural Database (CSD, Version 5.38, last update May 2017; Groom et al., 2016) for structures containing an 4-amino-3-methyl-1 $H$-1,2,4-triazole$5(4 H)$-thione moiety gave 69 hits; in 41 of these structures, the $\mathrm{C}=\mathrm{S}$ and/or $\mathrm{NH}_{2}$ groups are complexed with a metal ion. The 1,2,4-triazole ring is almost planar, with the largest deviation from the best plane through the ring atoms being $0.034 \AA$ [for the complex mer-trichlorido(dimethyl sulfoxide- $\kappa S)$ (4-amino3-ethyl-1,2,4- $\Delta^{2}$-triazoline-5-thione- $\kappa^{2} N, S$ )ruthenium(III) hemihydrate; CSD refcode KESQOO; Cingi et al., 2000].

\section{Synthesis and crystallization}

The reaction scheme used to synthesize the title compound, $\mathbf{3}$, is given in Fig. 6. Methyl 2-(thiophen-3-yl)acetate, 1, and 2-(thiophen-3-yl)acetohydrazide, 2 , were synthesized as described in a previous study (Vu Quoc et al., 2017).

For the synthesis of 4-amino-3-(thiophen-3-ylmethyl)- $1 \mathrm{H}$ 1,2,4-triazole-5(4H)-thione, $\mathbf{3}$, a mixture of hydrazide $\mathbf{2}$ $(5 \mathrm{mmol}), \mathrm{KOH}(0.01 \mathrm{~mol})$, ethanol $(10 \mathrm{ml})$ and carbon disulfide $(10 \mathrm{mmol})$ was stirred at room temperature until the formation of hydrogen sulfide stopped. An excess of alcohol was removed by distillation and the solid was washed with diethyl ether. A mixture of the resulting solid in water $(10 \mathrm{ml})$ and hydrazine hydrate $(15 \mathrm{ml})$ was then refluxed for $8 \mathrm{~h}$ at $353 \mathrm{~K}$. The reaction mixture was cooled and neutralized with dilute hydrochloric acid. The solid which precipitated was

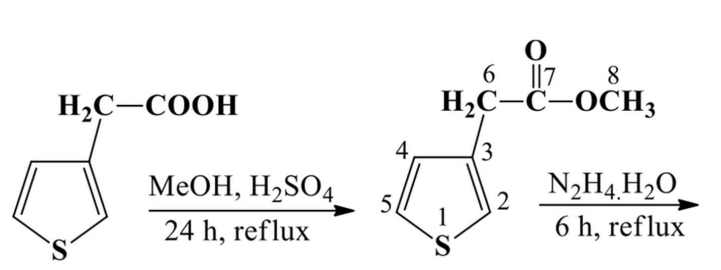

(1)

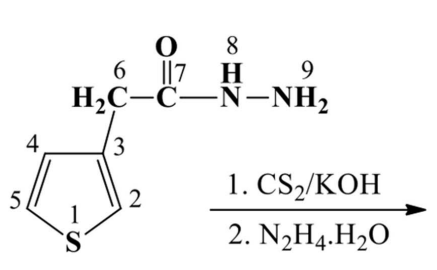

(2)

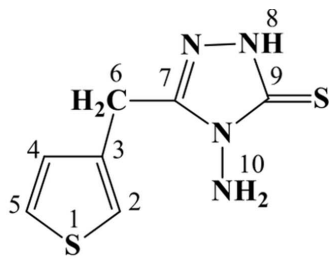

(3)

Figure 6

Reaction scheme for the title compound. 
filtered off, washed thoroughly with water, dried and recrystallized from an ethanol-water solvent mixture $(4: 1 \mathrm{v} / \mathrm{v})$ to give $0.63 \mathrm{~g}$ (yield $60.0 \%$ ) of $\mathbf{3}$ in the form of colourless crystals (m.p. 378 K). IR (Nicolet Impact 410 FT-IR, KBr, cm ${ }^{-1}$ ): 3452 $\left(v_{\mathrm{NH}}\right), 3088,2911\left(v_{\mathrm{CH}}\right), 1576\left(v_{\mathrm{C}=\mathrm{C}}\right.$ thiophene $), 1278,1207$ $\left(v_{\mathrm{C}=\mathrm{s}}\right) .{ }^{1} \mathrm{H}$ NMR [Bruker XL-500, $500 \mathrm{MHz}, d_{6}$-DMSO, $\delta$ $(\mathrm{ppm}), J(\mathrm{~Hz})]: 7.33\left(m, 1 \mathrm{H},{ }^{4} J=1.0, \mathrm{H}^{2}\right), 7.06\left(m, 1 \mathrm{H},{ }^{2} J=1.0\right.$, $\left.{ }^{5} J=5.0, \mathrm{H}^{4}\right), 7.49\left(d d, 1 \mathrm{H},{ }^{2} J=3.0,{ }^{4} J=5.0, \mathrm{H}^{5}\right), 4.04(s, 2 \mathrm{H}$, $\left.\mathrm{H}^{6}\right), 13.54\left(\mathrm{~s}, 1 \mathrm{H}, \mathrm{H}^{8}\right), 5.58\left(s, 2 \mathrm{H}, \mathrm{H}^{10}\right) .{ }^{13} \mathrm{C}$ NMR [Bruker XL$500,125 \mathrm{MHz}, d_{6}$-DMSO, $\left.\delta(\mathrm{ppm})\right]: 123.03$ (C2), 135.61 (C3), 128.98 (C4), 126.67 (C5), 25.60 (C6), 151.55 (C7), 166.47 (C9). Calculation for $\mathrm{C}_{7} \mathrm{H}_{8} \mathrm{~N}_{4} \mathrm{~S}_{2}: M=212$ a.u.

\section{Refinement}

Crystal data, data collection and structure refinement details are summarized in Table 2. Both thiophene rings are disordered over two orientations by a rotation of approximately $180^{\circ}$ around the $\mathrm{C} 5-\mathrm{C} 3$ bond. The final occupancy factors are 0.6957 (15) and 0.3043 (15). For the disordered thiophene ring, bond lengths and angles were restrained to the target mean values observed in $3-\mathrm{CH}_{2}$-thiophene fragments in the CSD (Groom et al., 2016) and the same anisotropic displacement parameters were used for equivalent atoms. The $\mathrm{H}$ atoms attached to atoms N1 and N4 were found in a difference density Fourier map and refined freely. The other $\mathrm{H}$ atoms were placed at calculated positions and refined in riding mode, with $\mathrm{C}-\mathrm{H}$ distances of 0.95 (aromatic) and $0.99 \AA\left(\mathrm{CH}_{2}\right)$, and isotropic displacement parameters equal to $1.2 U_{\mathrm{eq}}$ of the parent atoms. In the final cycles of refinement, two reflections showing very poor agreement were omitted as outliers.

\section{Funding information}

Funding for this research was provided by: VLIR-UOS (project No. ZEIN2014Z18 to LVM).

\section{References}

Bruker (2013). SAINT. Bruker AXS Inc., Madison, Wisconsin, USA. Bruker (2014). APEX2 and $S A D A B S$. Bruker AXS Inc., Madison, Wisconsin, USA.

Cingi, M. B., Lanfranchi, M., Pellinghelli, M. A. \& Tegoni, M. (2000). Eur. J. Inorg. Chem. pp. 703-711.

Dolomanov, O. V., Bourhis, L. J., Gildea, R. J., Howard, J. A. K. \& Puschmann, H. (2009). J. Appl. Cryst. 42, 339-341.

Groom, C. R., Bruno, I. J., Lightfoot, M. P. \& Ward, S. C. (2016). Acta Cryst. B72, 171-179.

McKinnon, J. J., Jayatilaka, D. \& Spackman, M. A. (2007). Chem. Commun. pp. 3814-3816.
Table 2

Experimental details.

\begin{tabular}{|c|c|}
\hline \multicolumn{2}{|l|}{ Crystal data } \\
\hline Chemical formula & $\mathrm{C}_{7} \mathrm{H}_{8} \mathrm{~N}_{4} \mathrm{~S}_{2}$ \\
\hline$M_{\mathrm{r}}$ & 212.29 \\
\hline Crystal system, space group & Monoclinic, $P 2_{1} / n$ \\
\hline Temperature (K) & 100 \\
\hline$a, b, c(\AA)$ & $7.6904(4), 13.0429(7), 9.0220(4)$ \\
\hline$\beta\left(^{\circ}\right)$ & $90.081(2)$ \\
\hline$V\left(\AA^{3}\right)$ & $904.95(8)$ \\
\hline$Z$ & 4 \\
\hline Radiation type & Mo $K \alpha$ \\
\hline$\mu\left(\mathrm{mm}^{-1}\right)$ & 0.54 \\
\hline Crystal size $(\mathrm{mm})$ & $0.32 \times 0.20 \times 0.08$ \\
\hline \multicolumn{2}{|l|}{ Data collection } \\
\hline Diffractometer & Bruker APEXII CCD \\
\hline Absorption correction & $\begin{array}{l}\text { Multi-scan (SADABS; Bruker, } \\
\text { 2014) }\end{array}$ \\
\hline$T_{\min }, T_{\max }$ & $0.710,0.746$ \\
\hline $\begin{array}{l}\text { No. of measured, independent and } \\
\text { observed }[I>2 \sigma(I)] \text { reflections }\end{array}$ & $27159,2784,2536$ \\
\hline$R_{\text {int }}$ & 0.025 \\
\hline$(\sin \theta / \lambda)_{\max }\left(\AA^{-1}\right)$ & 0.718 \\
\hline \multicolumn{2}{|l|}{ Refinement } \\
\hline$R\left[F^{2}>2 \sigma\left(F^{2}\right)\right], w R\left(F^{2}\right), S$ & $0.027,0.071,1.05$ \\
\hline No. of reflections & 2784 \\
\hline No. of parameters & 143 \\
\hline No. of restraints & 20 \\
\hline $\mathrm{H}$-atom treatment & $\begin{array}{l}\mathrm{H} \text { atoms treated by a mixture of } \\
\text { independent and constrained } \\
\text { refinement }\end{array}$ \\
\hline$\Delta \rho_{\max }, \Delta \rho_{\min }\left(\mathrm{e} \AA^{-3}\right)$ & $0.43,-0.35$ \\
\hline
\end{tabular}

Computer programs: APEX2 (Bruker, 2014), SAINT (Bruker, 2013), SHELXS97 (Sheldrick, 2008), SHELXL2014 (Sheldrick, 2015) and OLEX2 (Dolomanov et al., 2009).

Ming, L. S., Jamalis, J., Al-Maqtari, H. M., Rosli, M. M., Sankaranarayanan, M., Chander, S. \& Fun, H.-K. (2017). Chem. Data Collect. 9-10, 104-113.

Nguyen, N. L., Tran, T. D., Nguyen, T. C., Duong, K. L., Pfleger, J. \& Vu, Q. T. (2016). Vietnam. J. Chem. 54, 259-263.

Oyarce, J., Hernández, L., Ahumada, G., Soto, J. P., Valle, M. A., Dorcet, V., Carrillo, D., Hamon, J.-R. \& Manzur, C. (2017). Polyhedron, 123, 277-284.

Radi, S., Tighadouini, S., Bacquet, M., Degoutin, S., Dacquin, J.-P., Eddike, D., Tillard, M. \& Mabkho, Y. N. (2016). Molecules, 21, 888. Sheldrick, G. M. (2008). Acta Cryst. A64, 112-122.

Sheldrick, G. M. (2015). Acta Cryst. C71, 3-8.

Spackman, M. A. \& Jayatilaka, D. (2009). CrystEngComm, 11, 19-32. Vu, Q. T., Nguyen, N. L., Duong, K. L. \& Pfleger, J. (2016). Vietnam. J. Chem. 54, 730-735.

Vu Quoc, T., Nguyen Ngoc, L., Nguyen Tien, C., Thang Pham, C. \& Van Meervelt, L. (2017). Acta Cryst. E73, 901-904. 


\section{supporting information}

Acta Cryst. (2017). E73, 1389-1392［https://doi.org/10.1107/S2056989017012191]

Crystal structure of 4-amino-3-(thiophen-3-ylmethyl)-1 H-1,2,4-triazole-5(4H)thione

Trung Vu Quoc, Linh Nguyen Ngoc, Vy Do Truc, Linh Duong Khanh, Hung Ha Manh, Chien Thang Pham and Luc Van Meervelt

Computing details

Data collection: APEX2 (Bruker, 2014); cell refinement: SAINT (Bruker, 2013); data reduction: SAINT (Bruker, 2013); program(s) used to solve structure: SHELXS97 (Sheldrick, 2008); program(s) used to refine structure: SHELXL2014 (Sheldrick, 2015); molecular graphics: OLEX2 (Dolomanov et al., 2009); software used to prepare material for publication: OLEX2 (Dolomanov et al., 2009).

4-Amino-3-(thiophen-3-ylmethyl)-1H-1,2,4-triazole-5(4H)-thione

Crystal data

$\mathrm{C}_{7} \mathrm{H}_{8} \mathrm{~N}_{4} \mathrm{~S}_{2}$

$M_{r}=212.29$

Monoclinic, $P 2_{1} / n$

$a=7.6904$ (4) $\AA$

$b=13.0429$ (7) $\AA$

$c=9.0220(4) \AA$

$\beta=90.081(2)^{\circ}$

$V=904.95(8) \AA^{3}$

$Z=4$

\section{Data collection}

Bruker APEXII CCD diffractometer

$\varphi$ and $\omega$ scans

Absorption correction: multi-scan

(SADABS; Bruker, 2014)

$T_{\min }=0.710, T_{\max }=0.746$

27159 measured reflections

\section{Refinement}

Refinement on $F^{2}$

Least-squares matrix: full

$R\left[F^{2}>2 \sigma\left(F^{2}\right)\right]=0.027$

$w R\left(F^{2}\right)=0.071$

$S=1.05$

2784 reflections

143 parameters

20 restraints
$F(000)=440$

$D_{\mathrm{x}}=1.558 \mathrm{Mg} \mathrm{m}^{-3}$

Mo $K \alpha$ radiation, $\lambda=0.71073 \AA$

Cell parameters from 9996 reflections

$\theta=3.1-30.7^{\circ}$

$\mu=0.54 \mathrm{~mm}^{-1}$

$T=100 \mathrm{~K}$

Block, colorless

$0.32 \times 0.20 \times 0.08 \mathrm{~mm}$

2784 independent reflections

2536 reflections with $I>2 \sigma(I)$

$R_{\text {int }}=0.025$

$\theta_{\max }=30.7^{\circ}, \theta_{\min }=2.8^{\circ}$

$h=-11 \rightarrow 11$

$k=-18 \rightarrow 18$

$l=-12 \rightarrow 12$

Hydrogen site location: mixed

$\mathrm{H}$ atoms treated by a mixture of independent and constrained refinement

$w=1 /\left[\sigma^{2}\left(F_{\mathrm{o}}^{2}\right)+(0.0367 P)^{2}+0.3687 P\right]$ where $P=\left(F_{\mathrm{o}}^{2}+2 F_{\mathrm{c}}^{2}\right) / 3$

$(\Delta / \sigma)_{\max }=0.001$

$\Delta \rho_{\max }=0.43$ e $\AA^{-3}$

$\Delta \rho_{\min }=-0.35$ e $\AA^{-3}$ 


\section{Special details}

Geometry. All esds (except the esd in the dihedral angle between two 1.s. planes) are estimated using the full covariance matrix. The cell esds are taken into account individually in the estimation of esds in distances, angles and torsion angles; correlations between esds in cell parameters are only used when they are defined by crystal symmetry. An approximate (isotropic) treatment of cell esds is used for estimating esds involving l.s. planes.

Fractional atomic coordinates and isotropic or equivalent isotropic displacement parameters $\left(\AA^{2}\right)$

\begin{tabular}{llllll}
\hline & $x$ & $y$ & $z$ & $U_{\text {iso }} * / U_{\text {eq }}$ & Occ. $(<1)$ \\
\hline S1A & $0.63885(12)$ & $0.92996(9)$ & $-0.14786(11)$ & $0.02217(15)$ & $0.6957(15)$ \\
C1A & $0.7802(4)$ & $0.9045(2)$ & $0.0018(3)$ & $0.0237(6)$ & $0.6957(15)$ \\
H1A & 0.894252 & 0.931612 & 0.011838 & $0.028^{*}$ & $0.6957(15)$ \\
C2A & $0.7019(5)$ & $0.8398(5)$ & $0.1017(6)$ & $0.0164(5)$ & $0.6957(15)$ \\
H2A & 0.755868 & 0.816279 & 0.190184 & $0.020^{*}$ & $0.6957(15)$ \\
S1B & $0.7968(3)$ & $0.92242(15)$ & $-0.0160(2)$ & $0.02217(15)$ & $0.3043(15)$ \\
C1B & $0.6169(13)$ & $0.9240(9)$ & $-0.1362(11)$ & $0.0237(6)$ & $0.3043(15)$ \\
H1B & 0.606846 & 0.962783 & -0.224912 & $0.028^{*}$ & $0.3043(15)$ \\
C2B & $0.4838(15)$ & $0.8542(12)$ & $-0.0748(15)$ & $0.0164(5)$ & $0.3043(15)$ \\
H2B & 0.376467 & 0.840204 & -0.122989 & $0.020^{*}$ & $0.3043(15)$ \\
C3 & $0.52961(13)$ & $0.81192(7)$ & $0.05711(11)$ & $0.01448(18)$ & \\
C4A & $0.4817(8)$ & $0.8574(6)$ & $-0.0767(6)$ & $0.0211(5)$ & $0.6957(15)$ \\
H4A & 0.371471 & 0.847945 & -0.122328 & $0.025^{*}$ & $0.6957(15)$ \\
C4B & $0.6897(15)$ & $0.8412(12)$ & $0.1007(13)$ & $0.0211(5)$ & $0.3043(15)$ \\
H4B & 0.739939 & 0.817943 & 0.190900 & $0.025 *$ & $0.3043(15)$ \\
C5 & $0.40813(13)$ & $0.74501(8)$ & $0.14692(11)$ & $0.01518(18)$ & \\
H5A & 0.330663 & 0.706410 & 0.079246 & $0.018 *$ & \\
H5B & 0.476853 & 0.694874 & 0.204932 & $0.018^{*}$ & \\
C6 & $0.30116(12)$ & $0.80879(7)$ & $0.24957(10)$ & $0.01347(17)$ & \\
N3 & $0.14624(11)$ & $0.84574(7)$ & $0.22259(9)$ & $0.01560(16)$ & \\
N4 & $0.10978(11)$ & $0.90487(7)$ & $0.34611(9)$ & $0.01478(16)$ & \\
H4 & $0.011(2)$ & $0.9399(12)$ & $0.3552(18)$ & $0.030(4)^{*}$ & \\
C7 & $0.23720(12)$ & $0.90463(7)$ & $0.44686(10)$ & $0.01315(17)$ & \\
N2 & $0.36064(10)$ & $0.84243(6)$ & $0.38483(9)$ & $0.01294(15)$ & \\
S2 & $0.25221(3)$ & $0.96465(2)$ & $0.61239(3)$ & $0.01586(7)$ & \\
N1 & $0.52086(11)$ & $0.81396(7)$ & $0.44672(10)$ & $0.01611(17)$ & \\
H1C & $0.5008(19)$ & $0.7808(12)$ & $0.5337(17)$ & $0.023(4)^{*}$ & \\
H1D & $0.576(2)$ & $0.8692(13)$ & $0.4631(17)$ & $0.028(4)^{*}$ & \\
& & & & & \\
\end{tabular}

Atomic displacement parameters $\left(\AA^{2}\right)$

\begin{tabular}{lllllll}
\hline & $U^{11}$ & $U^{22}$ & $U^{33}$ & $U^{12}$ & $U^{13}$ & $U^{23}$ \\
\hline S1A & $0.0285(3)$ & $0.0166(2)$ & $0.0214(2)$ & $0.0005(2)$ & $0.0079(2)$ & $0.00146(16)$ \\
C1A & $0.0256(11)$ & $0.0213(13)$ & $0.0241(12)$ & $0.0030(9)$ & $-0.0027(9)$ & $0.0011(8)$ \\
C2A & $0.0091(10)$ & $0.0171(9)$ & $0.0229(9)$ & $-0.0038(9)$ & $0.0006(7)$ & $-0.0041(7)$ \\
S1B & $0.0285(3)$ & $0.0166(2)$ & $0.0214(2)$ & $0.0005(2)$ & $0.0079(2)$ & $0.00146(16)$ \\
C1B & $0.0256(11)$ & $0.0213(13)$ & $0.0241(12)$ & $0.0030(9)$ & $-0.0027(9)$ & $0.0011(8)$ \\
C2B & $0.0091(10)$ & $0.0171(9)$ & $0.0229(9)$ & $-0.0038(9)$ & $0.0006(7)$ & $-0.0041(7)$ \\
C3 & $0.0150(4)$ & $0.0134(4)$ & $0.0151(4)$ & $0.0023(3)$ & $0.0032(3)$ & $-0.0021(3)$
\end{tabular}




\begin{tabular}{lllllll} 
C4A & $0.0270(10)$ & $0.0233(12)$ & $0.0131(8)$ & $0.0096(8)$ & $0.0020(7)$ & $0.0019(7)$ \\
C4B & $0.0270(10)$ & $0.0233(12)$ & $0.0131(8)$ & $0.0096(8)$ & $0.0020(7)$ & $0.0019(7)$ \\
C5 & $0.0145(4)$ & $0.0144(4)$ & $0.0167(4)$ & $-0.0002(3)$ & $0.0023(3)$ & $-0.0024(3)$ \\
C6 & $0.0131(4)$ & $0.0131(4)$ & $0.0142(4)$ & $-0.0012(3)$ & $0.0011(3)$ & $0.0001(3)$ \\
N3 & $0.0140(4)$ & $0.0182(4)$ & $0.0146(4)$ & $0.0010(3)$ & $0.0005(3)$ & $-0.0022(3)$ \\
N4 & $0.0124(4)$ & $0.0169(4)$ & $0.0151(4)$ & $0.0027(3)$ & $0.0001(3)$ & $-0.0015(3)$ \\
C7 & $0.0122(4)$ & $0.0127(4)$ & $0.0145(4)$ & $0.0002(3)$ & $0.0019(3)$ & $0.0020(3)$ \\
N2 & $0.0105(3)$ & $0.0146(4)$ & $0.0138(3)$ & $0.0015(3)$ & $0.0000(3)$ & $0.0007(3)$ \\
S2 & $0.01442(12)$ & $0.01893(13)$ & $0.01423(12)$ & $0.00179(8)$ & $-0.00036(8)$ & $-0.00250(8)$ \\
N1 & $0.0113(4)$ & $0.0190(4)$ & $0.0181(4)$ & $0.0018(3)$ & $-0.0029(3)$ & $0.0020(3)$ \\
\hline
\end{tabular}

Geometric parameters $\left(A,{ }^{\circ}\right)$

\begin{tabular}{|c|c|c|c|}
\hline $\mathrm{S} 1 \mathrm{Aa}-\mathrm{C} 1 \mathrm{~A}$ & $1.763(4)$ & $\mathrm{C} 3-\mathrm{C} 4 \mathrm{~B}$ & $1.347(10)$ \\
\hline $\mathrm{C} 1 \mathrm{Aa}-\mathrm{H} 1 \mathrm{~A}$ & 0.9500 & $\mathrm{C} 3-\mathrm{C} 5$ & $1.5143(14)$ \\
\hline $\mathrm{C} 1 \mathrm{Aa}-\mathrm{C} 2 \mathrm{~A}$ & $1.375(5)$ & $\mathrm{C} 5-\mathrm{H} 5 \mathrm{~A}$ & 0.9900 \\
\hline $\mathrm{C} 2 \mathrm{Aa}-\mathrm{H} 2 \mathrm{~A}$ & 0.9500 & $\mathrm{C} 5-\mathrm{H} 5 \mathrm{~B}$ & 0.9900 \\
\hline $\mathrm{S} 1 \mathrm{Bb}-\mathrm{C} 1 \mathrm{~B}$ & $1.757(11)$ & $\mathrm{C} 5-\mathrm{C} 6$ & $1.4929(13)$ \\
\hline $\mathrm{C} 1 \mathrm{Bb}-\mathrm{H} 1 \mathrm{~B}$ & 0.9500 & $\mathrm{C} 6-\mathrm{N} 3$ & $1.3077(13)$ \\
\hline $\mathrm{C} 1 \mathrm{Bb}-\mathrm{C} 2 \mathrm{~B}$ & $1.478(12)$ & $\mathrm{C} 6-\mathrm{N} 2$ & $1.3745(12)$ \\
\hline $\mathrm{C} 2 \mathrm{Bb}-\mathrm{H} 2 \mathrm{~B}$ & 0.9500 & $\mathrm{~N} 3-\mathrm{N} 4$ & $1.3842(11)$ \\
\hline $\mathrm{C} 2 \mathrm{Aa}-\mathrm{C} 3$ & $1.431(3)$ & $\mathrm{N} 4-\mathrm{H} 4$ & $0.889(17)$ \\
\hline $\mathrm{C} 2 \mathrm{Bb}-\mathrm{C} 3$ & $1.357(11)$ & $\mathrm{N} 4-\mathrm{C} 7$ & $1.3356(12)$ \\
\hline $\mathrm{S} 1 \mathrm{Aa}-\mathrm{C} 4 \mathrm{~A}$ & $1.665(5)$ & $\mathrm{C} 7-\mathrm{N} 2$ & $1.3689(12)$ \\
\hline $\mathrm{C} 4 \mathrm{Aa}-\mathrm{H} 4 \mathrm{~A}$ & 0.9500 & $\mathrm{C} 7-\mathrm{S} 2$ & $1.6900(10)$ \\
\hline $\mathrm{S} 1 \mathrm{Bb}-\mathrm{C} 4 \mathrm{~B}$ & $1.707(11)$ & $\mathrm{N} 2-\mathrm{N} 1$ & $1.4021(11)$ \\
\hline $\mathrm{C} 4 \mathrm{Bb}-\mathrm{H} 4 \mathrm{~B}$ & 0.9500 & $\mathrm{~N} 1-\mathrm{H} 1 \mathrm{C}$ & $0.909(15)$ \\
\hline $\mathrm{C} 3-\mathrm{C} 4 \mathrm{~A}$ & $1.394(5)$ & $\mathrm{N} 1-\mathrm{H} 1 \mathrm{D}$ & $0.849(17)$ \\
\hline $\mathrm{C} 2 \mathrm{Aa}-\mathrm{C} 1 \mathrm{Aa}-\mathrm{S} 1 \mathrm{~A}$ & $110.3(3)$ & $\mathrm{C} 3-\mathrm{C} 2 \mathrm{Bb}-\mathrm{H} 2 \mathrm{~B}$ & 123.2 \\
\hline $\mathrm{C} 4 \mathrm{Aa}-\mathrm{S} 1 \mathrm{Aa}-\mathrm{C} 1 \mathrm{~A}$ & $92.56(19)$ & $\mathrm{C} 3-\mathrm{C} 5-\mathrm{H} 5 \mathrm{~B}$ & 109.5 \\
\hline $\mathrm{C} 2 \mathrm{Aa}-\mathrm{C} 1 \mathrm{Aa}-\mathrm{H} 1 \mathrm{~A}$ & 124.8 & $\mathrm{H} 5 \mathrm{~A}-\mathrm{C} 5-\mathrm{H} 5 \mathrm{~B}$ & 108.1 \\
\hline $\mathrm{S} 1 \mathrm{Aa}-\mathrm{C} 1 \mathrm{Aa}-\mathrm{H} 1 \mathrm{~A}$ & 124.8 & $\mathrm{C} 3-\mathrm{C} 4 \mathrm{Aa}-\mathrm{H} 4 \mathrm{~A}$ & 123.7 \\
\hline $\mathrm{C} 1 \mathrm{Aa}-\mathrm{C} 2 \mathrm{Aa}-\mathrm{H} 2 \mathrm{~A}$ & 123.9 & $\mathrm{C} 3-\mathrm{C} 4 \mathrm{Bb}-\mathrm{H} 4 \mathrm{~B}$ & 122.0 \\
\hline $\mathrm{C} 2 \mathrm{Bb}-\mathrm{C} 1 \mathrm{Bb}-\mathrm{S} 1 \mathrm{~B}$ & $107.9(7)$ & $\mathrm{C} 6-\mathrm{C} 5-\mathrm{C} 3$ & $110.59(8)$ \\
\hline $\mathrm{C} 4 \mathrm{Bb}-\mathrm{S} 1 \mathrm{Bb}-\mathrm{C} 1 \mathrm{~B}$ & $90.4(5)$ & $\mathrm{C} 6-\mathrm{C} 5-\mathrm{H} 5 \mathrm{~A}$ & 109.5 \\
\hline $\mathrm{S} 1 \mathrm{Bb}-\mathrm{C} 1 \mathrm{Bb}-\mathrm{H} 1 \mathrm{~B}$ & 126.1 & $\mathrm{C} 6-\mathrm{C} 5-\mathrm{H} 5 \mathrm{~B}$ & 109.5 \\
\hline $\mathrm{C} 2 \mathrm{Bb}-\mathrm{C} 1 \mathrm{Bb}-\mathrm{H} 1 \mathrm{~B}$ & 126.1 & $\mathrm{~N} 3-\mathrm{C} 6-\mathrm{C} 5$ & $126.34(9)$ \\
\hline $\mathrm{C} 1 \mathrm{Bb}-\mathrm{C} 2 \mathrm{Bb}-\mathrm{H} 2 \mathrm{~B}$ & 123.2 & $\mathrm{~N} 3-\mathrm{C} 6-\mathrm{N} 2$ & $110.46(8)$ \\
\hline $\mathrm{C} 1 \mathrm{Aa}-\mathrm{C} 2 \mathrm{Aa}-\mathrm{C} 3$ & $112.2(3)$ & $\mathrm{N} 2-\mathrm{C} 6-\mathrm{C} 5$ & $123.07(9)$ \\
\hline $\mathrm{S} 1 \mathrm{Aa}-\mathrm{C} 4 \mathrm{Aa}-\mathrm{H} 4 \mathrm{~A}$ & 123.7 & $\mathrm{C} 6-\mathrm{N} 3-\mathrm{N} 4$ & $103.94(8)$ \\
\hline $\mathrm{S} 1 \mathrm{Bb}-\mathrm{C} 4 \mathrm{Bb}-\mathrm{H} 4 \mathrm{~B}$ & 122.0 & $\mathrm{~N} 3-\mathrm{N} 4-\mathrm{H} 4$ & $122.3(11)$ \\
\hline $\mathrm{C} 4 \mathrm{Aa}-\mathrm{C} 3-\mathrm{C} 2 \mathrm{~A}$ & $112.3(3)$ & $\mathrm{C} 7-\mathrm{N} 4-\mathrm{N} 3$ & $113.41(8)$ \\
\hline $\mathrm{C} 4 \mathrm{Bb}-\mathrm{C} 3-\mathrm{C} 2 \mathrm{~B}$ & $112.1(6)$ & $\mathrm{C} 7-\mathrm{N} 4-\mathrm{H} 4$ & $124.3(11)$ \\
\hline $\mathrm{C} 4 \mathrm{Aa}-\mathrm{C} 3-\mathrm{C} 5$ & $123.1(2)$ & $\mathrm{N} 4-\mathrm{C} 7-\mathrm{N} 2$ & $103.39(8)$ \\
\hline $\mathrm{C} 2 \mathrm{Aa}-\mathrm{C} 3-\mathrm{C} 5$ & $124.6(2)$ & $\mathrm{N} 4-\mathrm{C} 7-\mathrm{S} 2$ & $130.50(8)$ \\
\hline $\mathrm{C} 2 \mathrm{Bb}-\mathrm{C} 3-\mathrm{C} 5$ & $122.9(4)$ & $\mathrm{N} 2-\mathrm{C} 7-\mathrm{S} 2$ & $126.10(7)$ \\
\hline $\mathrm{C} 4 \mathrm{Bb}-\mathrm{C} 3-\mathrm{C} 5$ & $124.8(4)$ & $\mathrm{C} 6-\mathrm{N} 2-\mathrm{N} 1$ & $124.07(8)$ \\
\hline
\end{tabular}




$\begin{array}{ll}\mathrm{C} 3-\mathrm{C} 5-\mathrm{H} 5 \mathrm{~A} & 109.5 \\ \mathrm{C} 3-\mathrm{C} 4 \mathrm{Aa}-\mathrm{S} 1 \mathrm{~A} & 112.6(3) \\ \mathrm{C} 3-\mathrm{C} 2 \mathrm{Aa}-\mathrm{H} 2 \mathrm{~A} & 123.9 \\ \mathrm{C} 3-\mathrm{C} 4 \mathrm{Bb}-\mathrm{S} 1 \mathrm{~B} & 115.9(7) \\ \mathrm{C} 3-\mathrm{C} 2 \mathrm{Bb}-\mathrm{C} 1 \mathrm{~B} & \\ & \\ \mathrm{C} 4 \mathrm{Aa}-\mathrm{S} 1 \mathrm{Aa}-\mathrm{C} 1 \mathrm{Aa}-\mathrm{C} 2 \mathrm{Aa} & 0.5(7) \\ \mathrm{S} 1 \mathrm{Aa}-\mathrm{C} 1 \mathrm{Aa}-\mathrm{C} 2 \mathrm{Aa}-\mathrm{C} 3 & -0.2(6) \\ \mathrm{C} 4 \mathrm{Bb}-\mathrm{S} 1 \mathrm{Bb}-\mathrm{C} 1 \mathrm{Bb}-\mathrm{C} 2 \mathrm{Bb} & -2.2(12) \\ \mathrm{S} 1 \mathrm{Bb}-\mathrm{C} 1 \mathrm{Bb}-\mathrm{C} 2 \mathrm{Bb}-\mathrm{C} 3 & 2.7(17) \\ \mathrm{C} 1 \mathrm{Bb}-\mathrm{C} 2 \mathrm{Bb}-\mathrm{C} 3-\mathrm{C} 4 \mathrm{Bb} & -1.8(18) \\ \mathrm{C} 1 \mathrm{Bb}-\mathrm{C} 2 \mathrm{Bb}-\mathrm{C} 3-\mathrm{C} 5 & 174.8(8) \\ \mathrm{C} 1 \mathrm{Aa}-\mathrm{C} 2 \mathrm{Aa}-\mathrm{C} 3-\mathrm{C} 4 \mathrm{Aa} & -0.3(6) \\ \mathrm{C} 1 \mathrm{Aa}-\mathrm{C} 2 \mathrm{Aa}-\mathrm{C} 3-\mathrm{C} 5 & -177.5(3) \\ \mathrm{C} 2 \mathrm{Aa}-\mathrm{C} 3-\mathrm{C} 4 \mathrm{Aa}-\mathrm{S} 1 \mathrm{Aa} & 0.7(7) \\ \mathrm{C} 5-\mathrm{C} 3-\mathrm{C} 4 \mathrm{Aa}-\mathrm{S} 1 \mathrm{Aa} & 178.0(2) \\ \mathrm{C} 1 \mathrm{Aa}-\mathrm{S} 1 \mathrm{Aa}-\mathrm{C} 4 \mathrm{Aa}-\mathrm{C} 3 & -0.7(5) \\ \mathrm{C} 2 \mathrm{Bb}-\mathrm{C} 3-\mathrm{C} 4 \mathrm{Bb}-\mathrm{S} 1 \mathrm{Bb} & 0.0(16) \\ \mathrm{C} 5-\mathrm{C} 3-\mathrm{C} 4 \mathrm{Bb}-\mathrm{S} 1 \mathrm{Bb} & -176.6(5) \\ \mathrm{C} 1 \mathrm{Bb}-\mathrm{S} 1 \mathrm{Bb}-\mathrm{C} 4 \mathrm{Bb}-\mathrm{C} 3 & 1.4(12) \\ \mathrm{C} 4 \mathrm{Bb}-\mathrm{C} 3-\mathrm{C} 5-\mathrm{C} 6 & 87.4(9) \\ \mathrm{C} 2 \mathrm{Bb}-\mathrm{C} 3-\mathrm{C} 5-\mathrm{C} 6 & -88.8(10) \\ \mathrm{C} 4 \mathrm{Aa}-\mathrm{C} 3-\mathrm{C} 5-\mathrm{C} 6 & -87.3(4) \\ \end{array}$

$\mathrm{C} 7-\mathrm{N} 2-\mathrm{C} 6$

$\mathrm{C} 7-\mathrm{N} 2-\mathrm{N} 1$

$\mathrm{N} 2-\mathrm{N} 1-\mathrm{H} 1 \mathrm{C}$

$\mathrm{N} 2-\mathrm{N} 1-\mathrm{H} 1 \mathrm{D}$

H1C $-\mathrm{N} 1-\mathrm{H} 1 \mathrm{D}$

$\mathrm{C} 2 \mathrm{Aa}-\mathrm{C} 3-\mathrm{C} 5-\mathrm{C} 6$

$\mathrm{C} 3-\mathrm{C} 5-\mathrm{C} 6-\mathrm{N} 3$

$\mathrm{C} 3-\mathrm{C} 5-\mathrm{C} 6-\mathrm{N} 2$

$\mathrm{N} 2-\mathrm{C} 6-\mathrm{N} 3-\mathrm{N} 4$

$\mathrm{C} 5-\mathrm{C} 6-\mathrm{N} 3-\mathrm{N} 4$

$\mathrm{C} 6-\mathrm{N} 3-\mathrm{N} 4-\mathrm{C} 7$

$\mathrm{N} 3-\mathrm{N} 4-\mathrm{C} 7-\mathrm{N} 2$

$\mathrm{N} 3-\mathrm{N} 4-\mathrm{C} 7-\mathrm{S} 2$

N4-C7- $2-\mathrm{C} 6$

$\mathrm{S} 2-\mathrm{C} 7-\mathrm{N} 2-\mathrm{C} 6$

$\mathrm{N} 4-\mathrm{C} 7-\mathrm{N} 2-\mathrm{N} 1$

$\mathrm{S} 2-\mathrm{C} 7-\mathrm{N} 2-\mathrm{N} 1$

$\mathrm{N} 3-\mathrm{C} 6-\mathrm{N} 2-\mathrm{C} 7$

$\mathrm{C} 5-\mathrm{C} 6-\mathrm{N} 2-\mathrm{C} 7$

$\mathrm{N} 3-\mathrm{C} 6-\mathrm{N} 2-\mathrm{N} 1$

$\mathrm{C} 5-\mathrm{C} 6-\mathrm{N} 2-\mathrm{N} 1$
$108.79(8)$

$127.13(8)$

108.7 (9)

$106.5(11)$

$109.7(14)$

$89.6(3)$

93.21 (12)

$-82.26(11)$

$0.31(11)$

$-175.64(9)$

$-0.17(11)$

$-0.04(11)$

$179.78(8)$

$0.23(10)$

$-179.61(7)$

$-178.40(9)$

$1.77(14)$

$-0.36(11)$

175.75 (9)

$178.32(9)$

$-5.57(14)$

Hydrogen-bond geometry $\left(A,{ }^{\circ}\right)$

$\mathrm{Cg} 3$ is the centroid of the $\mathrm{N} 2 / \mathrm{N} 3 / \mathrm{N} 4 / \mathrm{C} 6 / \mathrm{C} 7$ ring.

\begin{tabular}{lllll}
\hline$D-\mathrm{H} \cdots A$ & $D-\mathrm{H}$ & $\mathrm{H} \cdots A$ & $D \cdots A$ & $D-\mathrm{H}^{\cdots} A$ \\
\hline $\mathrm{N} 1-\mathrm{H} 1 C \cdots \mathrm{N} 3^{\mathrm{i}}$ & $0.909(15)$ & $2.622(15)$ & $3.3847(13)$ & $142.0(12)$ \\
$\mathrm{N} 1-\mathrm{H} 1 D^{\cdots} \mathrm{S} 2^{\mathrm{ii}}$ & $0.849(17)$ & $2.628(16)$ & $3.4163(9)$ & $154.9(13)$ \\
$\mathrm{N} 4-\mathrm{H} 4 \cdots \mathrm{S} 2^{\mathrm{iii}}$ & $0.890(16)$ & $2.395(15)$ & $3.2847(9)$ & $178.2(12)$ \\
$\mathrm{C} 1 B-\mathrm{H} 1 B^{\cdots} \cdot C g 3^{3 \mathrm{iv}}$ & 0.95 & 2.78 & $3.503(11)$ & 134 \\
\hline
\end{tabular}

Symmetry codes: (i) $x-1 / 2,-y+1 / 2, z-1 / 2$; (ii) $-x+1,-y+2,-z+1$; (iii) $-x,-y+2,-z+1$; (iv) $-x+1,-y+2,-z$. 